\title{
Les glaces polaires : une mémoire de l'évolution des gaz à effet de serre
}

\author{
D. Raynaud, J.-M. Barnola et J. Chappellaz \\ Laboratoire de Glaciologie et de Géophysique de l'Environnement du CNRS
}

\section{PRINCIPE DE BASE}

Les couches superficielles des calottes polaires, essentiellement en Antarctique et au Groenland, forment le névé polaire qui typiquement représente les 50 à 100 premiers mètres de la calotte. Elles sont caractérisées par une porosité ouverte sur l'atmosphère de surface qui, dans la partie basse du névé, va tendre à décroître avec la profondeur pour finalement produire un matériau à porosité totalement fermée: la glace polaire. La transformation progressive du névé en glace constitue la plus gigantesque expérience naturelle de frittage. Dans une première phase de la transformation, les cristaux de neige s'arrondissent pour produire les grains de névé. Ceux-ci vont ensuite progressivement se souder par transfert de matière sous l'effet de différents processus (sublimation-condensation, diffusion en surface ou volume) prenant place au cours de la densification contrôlée par le taux d'accumulation de la neige et par la température au sein du névé. En conséquence, les canaux intergranulaires vont se fermer bloquant tout échange avec l'atmosphère extérieure et, au moment où le dernier se ferme, environ $10 \%$ du volume de la glace ainsi formée est occupé par des pores (couramment dénommés les bulles de la glace) dans lesquels de l'air est emprisonné à une pression dépendant de la pression atmosphérique régnant à la surface de la calotte. L'air de la glace ayant été isolé dans les couches les plus profondes du névé est donc plus jeune que la glace elle-même.

Si l'air se trouvant dans les canaux du névé en communication avec la surface est mélangé par convection avec l'air extérieur, alors sa composition est celle de l'atmosphère de surface. Ceci est, en fait, uniquement vrai au sein des couches les plus superficielles. Plus en profondeur dans le névé, le long de la colonne non soumise à la convection, le mélange ne s'effectue que par diffusion, ce qui prend un certain temps (typiquement une dizaine d'années), et un fractionnement se produit dû à l'effet de la gravité avec pour conséquence une concentration plus élevée des molécules gazeuses les plus lourdes vers le bas du névé juste avant le piégeage définitif de l'air dans les bulles de la glace. La correction à apporter, liée à cet effet de gravité, est faible et calculable avec une précision suffisante. En résumé, la composition de l'air initialement incorporée dans la glace et une fois corrigée de la gravitation est celle régnant en surface une dizaine d'années avant le piégeage par la glace.

Après avoir piégé l'air, la glace se densifie avec la profondeur, ce qui conduit à une réduction progressive du volume des bulles et à une augmentation concomitante de la pression du gaz atmosphérique en leur sein. Sous plusieurs centaines de mètres d'accumulation de glace, les molécules de gaz ont tendance à être progressivement encagées au sein même des molécules $\mathrm{H}_{2} \mathrm{O}$ de la glace pour former ce que l'on appelle des clathrates. Passé une certaine profondeur, qui dépend de la température et de la pression, la totalité de l'air est intimement incorporé sous forme de clathrate et la glace à sa sortie du forage est parfaitement translucide, sans bulle visible. Les analyses d'air de même âge mais extrait de glaces correspondant à des étapes différentes de densification de la glace indiquent que, moyennant certaines précautions expérimentales, la composition de l'air extrait n'est pas influencée par les processus prenant place au cours de la densification. Par contre des réactions chimiques prenant place dans de la glace à taux élevé d'impuretés, comme au Groenland, peut altérer la composition en $\mathrm{CO}_{2}$ de l'air contenu dans la glace. C'est pourquoi les variations passées du gaz carbonique sont actuellement extraites essentiellement des archives antarctiques.

\section{II $\square$ L'ENREGISTREMENT GLACIAIRE DES GAZ À EFFET DE SERRE}

L'échantillonnage en continu de l'air atmosphérique polaire dans les couches proches de la surface en Antarctique et au Groenland autorise l'extension au passé et de façon particulièrement directe de l'enregistrement instrumental de traces gazeuses à effet de serre critiques comme le $\mathrm{CO}_{2}$ ou le $\mathrm{CH}_{4}$ et obtenu en différentes stations du globe au cours des dernières décades. L'enregistrement de l'air piégé dans la glace permet en particulier de documenter la période fortement perturbée par l'activité humaine (les derniers siècles) et non couverte par l'enregistrement instrumental atmosphérique, la période pré-industrielle (le dernier millénaire), et à plus long terme l'échelle des cycles glaciaire-interglaciaires (échelle de la centaine de milliers d'années ou plus). Ces différentes échelles de temps nous permettent de mettre en perspective l'influence anthropogénique et d'appréhender les interactions entre variabilité ou changements climatiques et gaz à effet de serre.

\subsection{L'ère industrielle}

La figure $I$ illustre l'enregistrement du $\mathrm{CO}_{2}$ et du $\mathrm{CH}_{4}$ au cours des deux derniers siècles. Elle montre le bon raccordement entre les mesures effectuées dans la glace 
(points et carrés) et les enregistrements instrumentaux mesurés directement dans l'atmosphère (traits pleins). Elle indique de façon marquée l'augmentation de type exponentiel de ces traces gazeuses radiativement actives au cours de la période industrielle. Les concentrations en gaz carbonique et méthane ont ainsi crû respectivement d'environ 27 et $145 \%$ depuis l'époque pré-industrielle. De telles augmentations sont essentiellement liées à la combustion des énergies fossiles et aux modifications anthropiques des écosystèmes dans le cas du $\mathrm{CO}_{2}$ et aux émissions à partir des rizières, des ruminants, des décharges, de la combustion de la biomasse et de la production et de l'utilisation d'énergies fossiles en ce qui concerne le $\mathrm{CH}_{4}$. L'évolution du $\mathrm{N}_{2} \mathrm{O}$ (autre trace gazeuse radiativement active), bien que moins bien documentée, révèle elle aussi une augmentation de la teneur atmosphérique (d'environ $13 \%)$ depuis l'époque pré-industrielle.

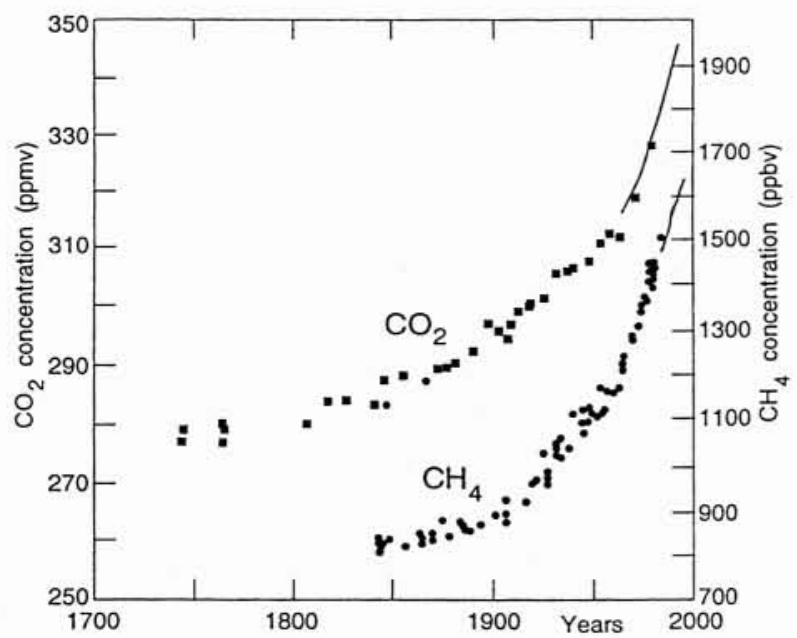

1. Evolution du $\mathrm{CO}_{2}$ et du $\mathrm{CH}_{4}$ au cours des deux derniers siècles à partir de l'analyse des bulles d'air de la glace (carrés et points) et des enregistrements atmosphériques (traits pleins). (D'après Friedli et al. 1966 ; Etheridge et al. 1992).

\subsection{Le dernier millénaire}

Les archives glaciaires permettent aussi de documenter la variabilité des traces gazeuses à effet de serre durant la partie du dernier millénaire ayant précédé l'ère industrielle et au cours de laquelle le climat était proche de l'actuel, avec cependant des fluctuations climatiques plus ou moins globales et d'assez faible amplitude (de l'ordre du degré au moins) : le petit âge glaciaire et le réchauffement médiéval. $\mathrm{Le} \mathrm{CO}_{2}$ indique un niveau moyen pré-industriel de 280 ppmv (parties par million en volume) avec des variations dont l'amplitude maximale est d'environ $10 \mathrm{ppmv}$. Quant au méthane on observe des variations de l'ordre de $10 \%$ autour d'un niveau pré-industriel moyen d'environ 700 ppbv (parties par milliard en volume). L'origine de ces variations de faible amplitude n'est pas clairement établie. Elles peuvent être la conséquence de l'impact des fluctuations climatiques précédemment citées (petit âge glaciaire, ...) sur les sources et puits naturels de $\mathrm{CO}_{2}$ et $\mathrm{CH}_{4}$.

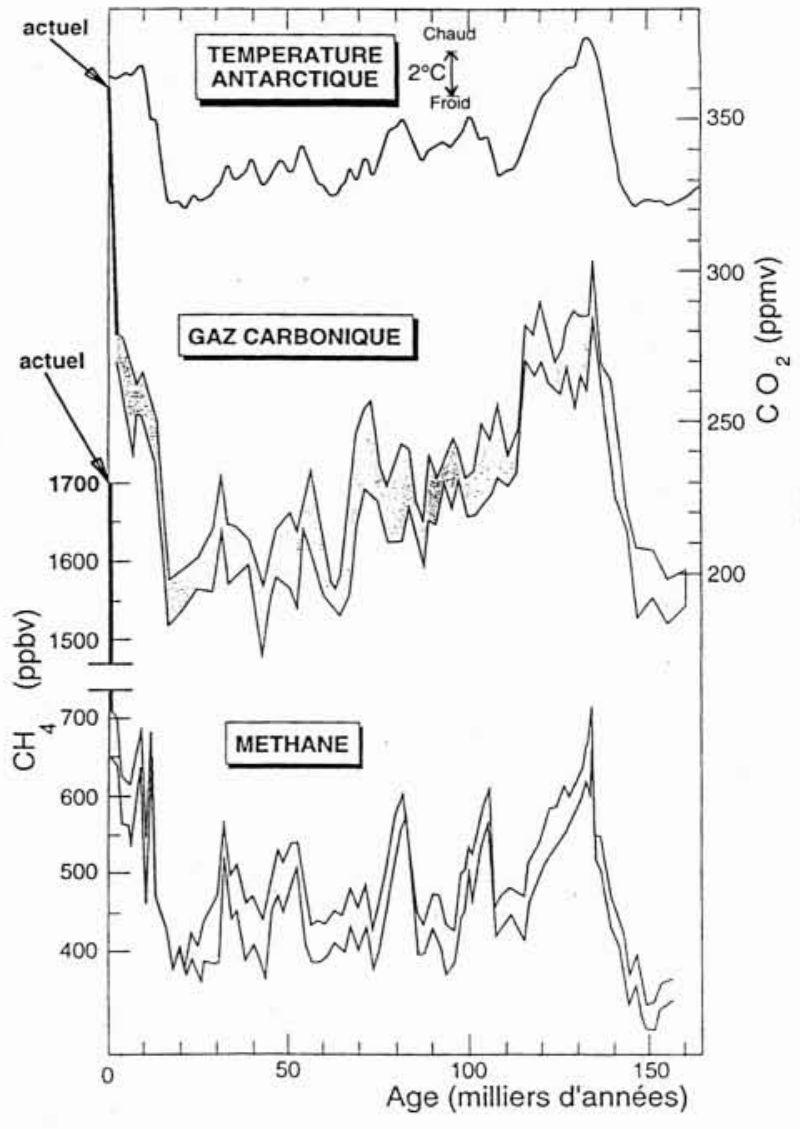

2. Variations du $\mathrm{CO}_{2}$ et du $\mathrm{CH}_{4}$ atmosphérique au cours du dernier cycle climatique à partir de l'analyse de la carotte de Vostok (Antarctique). Comparaison avec les concentrations actuelles. (D'après Barnola et al., $1987\left(\mathrm{CO}_{2}\right)$; Chappellaz et al., $1990\left(\mathrm{CH}_{4}\right)$; Jouzel et al., 1987 (température).

Cependant on ne peut écarter a priori l'influence de la variabilité naturelle à plus hautes fréquences du système climatique sur ces mêmes sources et puits ou encore celle d'un impact anthropique non négligeable avant l'ère préindustrielle. L'extension de l'enregistrement à l'ensemble de l'Holocène (les derniers 10000 ans) devrait permettre de préciser les causes de la variabilité du $\mathrm{CO}_{2}$ et du $\mathrm{CH}_{4}$ sous des conditions climatiques proches de celles que nous connaissons actuellement.

\subsection{Le cycle glaciaire-interglaciaire}

Durant les grands cycles climatiques du dernier million d'années le climat de la Terre a oscillé entre des modes glaciaires et interglaciaires avec des variations de la température à l'échelle globale de l'ordre de $5^{\circ} \mathrm{C}$. Que s'est-il passé au niveau des traces gazeuses radiativement actives lors de ces grands changements climatiques ? Les données existantes provenant des archives contenues dans les carottes de glace couvrent les deux derniers cycles ( 250000 ans) pour le $\mathrm{CO}_{2}$ et le $\mathrm{CH}_{4}$. La figure 2 montre les profils uniques obtenus sur l'ensemble du premier cycle climatique à partir de la carotte antarctique de Vostok. On 
a aussi positionné sur la figure les concentrations actuelles, ce qui permet de mettre en perspective l'impact spectaculaire de l'activité humaine au cours des derniers siècles. On a enfin porté sur la même figure, comme référence climatique, la variation de la température au-dessus de la station Vostok telle qu'elle est obtenue à partir de la composition isotopique de la glace mesurée sur la même carotte de Vostok. Les variations observées jusqu'à l'époque pré-industrielle indiquent des concentrations en $\mathrm{CO}_{2}$ et $\mathrm{CH}_{4}$ oscillant respectivement entre approximativement 200 et $300 \mathrm{ppmv}$ et 300 et $700 \mathrm{ppvb}$, avec les valeurs maximales obtenues durant les interglaciaires chauds et les minimales correspondant aux conditions glaciaires les plus rigoureuses. Il existe de façon générale une forte corrélation entre les traces gazeuses à effet de serre d'une part et la température de l'autre, ce qui pose la question de la contribution des gaz à effet de serre aux changements climatiques au cours d'un cycle glaciaire-interglaciaire. Il faut en premier lieu noter que les variations du gaz carbonique et du méthane ont dû être initiées à ces échelles temporelles à partir de modifications des réservoirs clés que constituent l'océan (pour le $\mathrm{CO}_{2}$ ) et les écosystèmes continentaux (en particulier dans le cas du méthane), le moteur au point de départ pouvant être par exemple un changement de la circulation atmosphérique et océanique stimulé par un forçage externe au système : les variations orbitales qui jouent sur les variations et la répartition de l'insolation. Si le moteur astronomique peut être à l'origine des grands changements glaciaires-interglaciaires, il ne peut cependant expliquer à lui seul qu'une faible partie de l'amplitude du cycle dominant de 100000 ans observé dans l'enregistrement paléoclimatique. La découverte de variations majeures du $\mathrm{CO}_{2}$ et du $\mathrm{CH}_{4}$ corrélées à celles du climat accrédite l'idée que ces gaz à effet de serre ont pu jouer un rôle important d'amplificateur des variations climatiques. Différentes approches indépendantes, incluant l'utilisation de modèles statistiques simples (consistant à comparer les courbes de paléotempératures aux différents forçages susceptibles de les expliquer) et des simulations climatiques à partir de modèles de circulation générale ont été utilisées pour évaluer ce rôle amplificateur. Elles conduisent à attribuer aux gaz à effet de serre entre 40 et $50 \%$ des effets de température observées. Ainsi l'augmentation des gaz à effet de serre expliquerait jusqu'à 2 des 4 à $5{ }^{\circ} \mathrm{C}$ du réchauffement moyen de la Terre ayant typiquement pris place au cours d'une déglaciation. 